\title{
Site-Specific Chemical Modification of Human Serum Albumin with Polyethylene Glycol Prolongs Half-life and Improves Intravascular Retention in Mice
}

\author{
Ting Zhao, ${ }^{a}$ Yan-Na Cheng, ${ }^{b}$ Hai-Ning Tan, ${ }^{c}$ Jin-Feng Liu, ${ }^{a}$ Huan-Li Xu, ${ }^{a}$ Guang-Li Pang, ${ }^{d}$ and \\ Feng-Shan Wang*,a,c \\ ${ }^{a}$ Institute of Biochemical and Biotechnological Drugs, School of Pharmaceutical Sciences, Shandong \\ University; ${ }^{b}$ Department of Pharmacology, School of Pharmaceutical Sciences, Shandong University; ${ }^{c}$ National \\ Glycoengineering Research Center, Shandong University; Jinan 250012, China: and ${ }^{d}$ Shandong Taibang Biological \\ Products Co., Ltd.; Taian 271000, China. \\ Received April 29, 2011; accepted November 22, 2011; published online December 21, 2011
}

\begin{abstract}
Human serum albumin (HSA) is used as an important plasma volume expander in clinical practice. However, the infused HSA may extravasate into the interstitial space and induce peripheral edema in treating the critical illness related to marked increase in capillary permeability. Such poor intravascular retention also demands a frequent administration of HSA. We hypothesize that increasing the molecular weight of HSA by PEGylation may be a potential approach to decrease capillary permeability of HSA. In the present study, HSA was PEGylated in a site-specific manner and the PEGylated HSA carrying one chain of polyethylene glycol (PEG) $(20 \mathrm{kDa})$ per HSA molecule was obtained. The purity, PEGylated site and secondary structure of the modified protein were characterized by sodium dodecyl sulfate polyacrylamide gel electrophoresis (SDS-PAGE), thiol group blockage method and circular dichroism (CD) measurement, respectively. In addition, the pharmacokinetics in normal mice was investigated, vascular permeability of the PEGylated HSA was evaluated in lipopolysaccharide (LPS)-induced lung injury mouse model and the pharmacodynamics was investigated in LPS-induced sepsis model with systemic capillary leakage. The results showed that the biological half-life of the modified HSA was approximately 2.3 times of that of the native HSA, PEG-HSA had a lower vascular permeability and better recovery in blood pressure and haemodilution was observed in rats treated with PEG-HSA. From the results it can be inferred that the chemically well-defined and molecularly homogeneous PEGylated HSA is superior to HSA in treating capillary permeability increase related illness because of its longer biological half-life and lower vascular permeability.
\end{abstract}

Key words human serum albumin; PEGylation; biological half-life; capillary permeability

Human serum albumin (HSA), the most abundant plasma protein, accounts for about $60 \%$ of the total protein in serum and typically presents at a concentration of $40 \mathrm{mg} / \mathrm{mL}$. It consists of a single non-glycosylated polypeptide chain of about 585 amino acid residues forming a heart-shaped molecule with a molecular weight of $66.5 \mathrm{kDa} .{ }^{1)}$ It contains 35 cysteine residues, forming 17 disulphide bridges, with one free thiol at Cys34. ${ }^{2)}$

HSA has numerous physiological functions, including regulating the distribution of water, maintaining normal plasma colloid oncotic pressure (COP) and transporting endogenous and exogenous ligands. ${ }^{3)}$ Clinically, HSA is mainly used in pharmaceutical preparations for the treatment of hypovolemia caused by traumatic shock, massive hemorrhage, burns, cirrhosis with ascites and hypoalbuminemia by maintaining COP and increasing the circulating plasma volume, with a usual dosage of $10 \mathrm{~g} /$ dose or more. ${ }^{4)}$

HSA is most commonly isolated by fractionation of plasma obtained from blood donors. However, since the supply for material plasma is limited in the global range, the shortage of donor blood has become a limiting factor for the production of HSA preparation. Aiming at this issue, many efforts have been made to develop a substitute for the plasma-derived HSA (pdHSA) by means of gene manipulation techniques. However, due to the low production yields and the high clinical dosage of recombinant HSA (rHSA), there is still an unsettled problem concerning the establishment of techniques for high purity, low cost, industrial large-scale production of rHSA.,4)
Therefore, for time being, the main use of rHSA will not replace the pdHSA in clinical application. In another word, plasma will still be the most important source of HSA as a plasma volume expander.

In terms of the clinical application of HSA in maintaining COP and increasing the plasma volume, HSA was developed for the treatment for shocks, burns, trauma, and various critical illness that result in albumin loss. It is well known that increased microvascular permeability is an important pathphysiological alteration in diseases such as burn injury, sepsis and trauma, and often develop transcapillary albumin leakage, hypovolemia, and interstitial edema. ${ }^{5,6}$ Under this condition, correction of low plasma volume therefore becomes essential. However, the plasma expanding effect of HSA is transient due to a continuous leakage of macromolecules into the interstitial space, ${ }^{7)}$ such poor intravascular retention does not only lead to interstitial edema but also demands a frequent administration of HSA infusion to maintain the desired blood concentration. ${ }^{8)}$

We hypothesized whether an albumin alternative agent with the properties of good intravascular retention and poor extravasation could be developed to solve the problems of interstitial edema and frequent infusion. Therefore, increasing the molecular weight of HSA may be a very potential approach to make modified HSA as a superior volume expander. Covalent attachment of polyethylene glycol (PEG) to proteins, namely PEGylation offers us a possibility to design and obtain such a modified HSA. The linkage of the PEG may endow HSA with bigger molecular size and larger hydrodynamic radius and 
thus reduce the extravasation. ${ }^{9,10)}$ This type of albumin could not only prevent the interstitial edema but also reduce the infusion frequency, which is valuable to decrease the mount required in clinical use indirectly and further alleviates the problem of short supply of plasma donor.

In the present study, we PEGylated HSA in a site-specific manner by taking advantage of the unusual chemical reactivity of the only one free Cys34 of the HSA molecule and the high specificity of $\mathrm{PEG}_{20 \mathrm{kDa}}$-maleimide for the protein - $\mathrm{SH}$ groups. This site-specific PEGylation targeting the unique free Cys34 could generate chemically well-defined and molecularly homogeneous modified HSA product and may be also useful in preventing dimerization. The pdHSA was PEGylated, purified and the purity, PEGylation site and secondary structure was generally characterized. In addition, the circulation halflife in blood was determined using a mouse model system, the vascular permeability was evaluated in a murine model of LPS-induced lung injury and the pharmacodynamics of PEGylated HSA was investigated in LPS-induced sepsis model with systemic capillary leakage.

\section{MATERIALS AND METHODS}

Materials DEAE Sepharose Fast Flow and Sephacryl S-200 were purchased from GE Healthcare, U.S.A. Maleimide derivatized $\mathrm{PEG}_{20 \mathrm{kDa}}\left(\mathrm{PEG}_{20 \mathrm{kDa}}-\mathrm{Mal}\right)$ was purchased from Beijing Kaizheng Biotech Co., Ltd., China. HSA was kindly provided by Shandong Taibang Biologic Products, Inc., China. Iodine-125 $\left({ }^{125} \mathrm{I}\right)$ was purchased from China Institute of Atomic Energy, China. Lipopolysaccharide (LPS) was the product of Sigma (from Escherichia coli 055:B5), U.S.A., and other reagents and chemicals were of analytical grade commercially available. The circular dichroism (CD) spectra were measured using an Applied Photophysics Chirascan spectropolarimeter, U.K. Dynamic light scattering measurements were performed on a multi-angle laser photometer (DAWN HELEOS, U.S.A.). Histology examination was conducted with inverted fluorescence microscope (Olympus, Japan). The haematocrit was measured on automatic hematology analyzer (Sysmex, Japan). The arterial pressure was monitored on the BL-420F Data Acquisition and Analysis System (ChengDu Technology and Market Co., Ltd., China).

Animals Four to five week-old male Kunming mice weighing $18-20 \mathrm{~g}$ and adult male Wistar rats weighing 300 $380 \mathrm{~g}$, offered by experimental animal center of Shandong University, were used in the experiments. The animals were housed in animal facilities accredited by the Shandong Council on Animal Care and treated in accordance with approved protocols. Animals were maintained in a specific pathogen-free environment that was temperature-controlled $\left(23 \pm 2^{\circ} \mathrm{C}\right)$ and humidity-controlled $(60 \pm 10 \%)$, under a $12: 12 \mathrm{~h}$ light/dark cycle.

PEGylation of HSA with PEG $_{\mathbf{2 0} \mathbf{k D a}}$-Mal HSA was purified by Sephacryl S-200 gel filtration and ultrafiltration employing a membrane with $50 \mathrm{kDa}$ cut-off. Fraction containing HSA was pooled and lyophilized.

The optimal reaction conditions of PEGylation of HSA with $\mathrm{PEG}_{20 \mathrm{kDa}}-\mathrm{Mal}$ were studied. The effects of buffer $\mathrm{pH}$, protein/polymer molar ratio and reaction time on the modification rates and homogenicity of products were investigated by single factor analysis. $\mathrm{PEG}_{20 \mathrm{kDa}}$-Mal was added to HSA solu- tions $(10 \mathrm{mg} / \mathrm{mL})$ in $50 \mathrm{mmol} / \mathrm{L}$ phosphate, $10 \mathrm{mmol} / \mathrm{L}$ ethylene diaminetetraacetic acid (EDTA) with different $\mathrm{pH}$ values of $6.0,6.5$, and 7.0, to obtain different protein/polymer molar ratios of $1: 1,1: 2,1: 3,1: 5,1: 10$, and $1: 15$. The reaction mixtures were incubated at $37^{\circ} \mathrm{C}$ for $3,5,10,15,20$, and $25 \mathrm{~h}$, respectively. Each PEGylation reaction was quenched by diluting the mixture 10 -fold with reaction buffer. Sodium dodecyl sulfate-polyacrylamide gel electrophoresis (SDS-PAGE) was used to assess the modification rates and homogenicity of the final reaction mixtures. The ratios of the optical densities of the PEG-HSA bands and the HSA bands represented the modification rates of products under different reaction conditions.

Purification of the PEGylated HSA The PEGylated HSA was seperated from the unreacted HSA and $\mathrm{PEG}_{20 \mathrm{kDa}}-\mathrm{Mal}$ by anion exchange chromatography on a $2.6 \mathrm{~cm} \times 20 \mathrm{~cm}$ DEAE Sepharose FF column at room temperature. The reaction mixture was dialyzed against $10 \mathrm{mmol} / \mathrm{L}$ phosphate buffer, $\mathrm{pH} 6.5$ (buffer A), then applied to the column and washed thoroughly with buffer A until no protein was eluted, and the column was eluted with a linear gradient of $0.1-0.3 \mathrm{~mol} / \mathrm{L} \mathrm{NaCl}$ in buffer A over 5 column volume. Peaks corresponding to monoPEGylated protein and unreacted HSA were separately pooled and concentrated by ultrafiltration. Fractions were anaylzed by $10 \%$ SDS-PAGE.

Purity identification of PEGylated HSA by SDS-PAGE Reaction mixtures under different reaction conditions and fractions from DEAE sepharose FF column were analyzed by SDS-PAGE. A discontinuous system was employed. ${ }^{11)}$ The gels consisted of a separating gel containing $10 \%(\mathrm{w} / \mathrm{v})$ acrylamide and a stacking gel containing $3 \%(\mathrm{w} / \mathrm{v})$ acrylamide. Dimensions of the gel immersed in running buffer $(0.1 \%$ SDS, $0.05 \mathrm{~mol} / \mathrm{L}$ Tris $/ 0.38 \mathrm{~mol} / \mathrm{L}$ glycine buffer, $\mathrm{pH}$ $8.3)$ were $8 \mathrm{~cm} \times 8 \mathrm{~cm} \times 0.75 \mathrm{~mm}$. Samples in loading buffer (2\% SDS, 5\% mercaptoethanol, 25\% glycerol, $0.01 \%$ bromophenol blue, $0.01 \mathrm{~mol} / \mathrm{L}$ Tris- $\mathrm{HCl}, \mathrm{pH} 8.8$ ) were incubated at $100^{\circ} \mathrm{C}$ for $5 \mathrm{~min}$ before electrophoresis. Electrophoresis was programmed to a two-step mode with applying constant voltages of $80 \mathrm{~V}$ in stacking gel and $130 \mathrm{~V}$ in the separation gel. Coomassie brilliant blue and barium iodide staining was used to detect HSA and PEG molecules (free and conjugated), respectively. ${ }^{12)}$ Firstly, the gel was dipped in $40 \mathrm{~mL}$ of $2 \mathrm{~mol} / \mathrm{L}$ perchloric acid. After $10 \mathrm{~min}, 10 \mathrm{~mL}$ of $5 \% \mathrm{BaCl}_{2}$ and $2 \mathrm{~mL}$ of $0.1 \mathrm{~mol} / \mathrm{L}$ iodine solution were added. Bands corresponding to PEGylated conjugates and free PEG appeared brown color. Whereafter, the gel was rinsed with distilled water and stained with Coomassie brilliant.

Determination of PEGylation Site To determine whether the activated PEG was specifically attached to Cys34 of the HSA molecule, HSA was previously incubated with $N$-ethylmaleimide (NEM), a reagent that specifically and covalently binds to thiol residues, for $5 \mathrm{~h}$ at $37^{\circ} \mathrm{C}$. And then PEG-Mal was added to reaction solution and incubated under the optimal PEGylation reaction conditions. ${ }^{13)}$ SDS-PAGE was subsequently employed to reveal whether NEM-treated HSA could be PEGylated.

Secondary Structure Analysis by Far-UV CD Measurement The secondary structure of HSA and PEGHSA were analyzed by CD spectra. The CD spectra were recorded in the far UV $(190-260 \mathrm{~nm})$ using $5 \mu \mathrm{mol} / \mathrm{L} \mathrm{HSA}$ solution or equimolar quantity of PEGylated HSA in $50 \mathrm{mmol} / \mathrm{L}$ phosphate buffer, $\mathrm{pH} 7.4$ at room temperature. ${ }^{8)}$ The band- 
width is $1 \mathrm{~nm}$ and time-per-point is $1 \mathrm{~s}$. Protein concentration was measured using Lowry method. The analysis was carried out using the Pro-Data Viewer software.

Hydrodynamic Radius Determination by Dynamic Light Scattering The molecular Hydrodynamic radii $\left(R_{\mathrm{h}}\right)$ of HSA and PEG-HSA were determined by dynamic light scattering (DLS) measurements. DLS measurements were performed on a multi-angle laser photometer equipped with a linearly polarized gallium arsenide (GaAs) laser $(\lambda=658 \mathrm{~nm})$, which were conducted at a scattering angle of $99^{\circ}$. The protein samples were prepared by dissolution of an appropriated amount of freeze-dried powder in saline and the protein concentration was determined by bicinchoninic acid assay. The $R_{\mathrm{h}}$ is calculated by using the Stokes-Einstein equation: $R_{\mathrm{h}}=k_{\mathrm{B}} T / 6 \pi \eta_{0} D$ where $R_{\mathrm{h}}$ is the hydrodynamic radius, $k_{\mathrm{B}}$ is Boltzmann's constant, $T$ is the absolute temperature and $\eta_{0}$ is the solvent viscosity, $D$ is the translational diffusion coefficient. ${ }^{14)}$

Pharmacokinetics of Native and PEGylated HSA HSA and PEG-HSA were labeled with ${ }^{125}$ I by iodogen (1,3,4,6-tetrachoro-3a,6a-diphenylglycoluri) methods as described previously. ${ }^{15)}$ Briefly, $50 \mu \mathrm{g}$ protein was incubated with $0.5 \mathrm{mCi}$ of carrier-free $\mathrm{Na}^{125} \mathrm{I}$ in a calibrated iodogen reaction vial with gentle shaking for $30 \mathrm{~min}$ at room temperature. Then the reaction was stopped by adding $150 \mu \mathrm{L}$ of $50 \mathrm{mmol} / \mathrm{L}$ PBS, pH 7.4. Purification of the labeled protein was performed by gel filtration on Sephadex G-25 column, previously blocked with $1 \%$ bovine serum albumin solution and equilibrated with sterile and pyrogen-free $10 \mathrm{mmol} / \mathrm{L} \mathrm{PBS}, \mathrm{pH}$ 7.4. The flow rate was $0.3 \mathrm{~mL} / \mathrm{min}$. Fractions were detected by activity measurement in a gamma counter, three milliliter fractions of the highest concentrations of the labeled proteins were collected and pooled.

Single dose pharmacokinetics studies were conducted in Kunming mice. ${ }^{125} \mathrm{I}-\mathrm{HSA}$ and ${ }^{125} \mathrm{I}-\mathrm{PEG}-\mathrm{HSA}$ in saline were injected into the tail vein of kunming mice, blood $(100 \mu \mathrm{L})$ samples were periodically collected into heparinized needle at 5, 20, $45 \mathrm{~min}, 1,4,8,12,18,24,36,48,60,72,84$, and $96 \mathrm{~h}$ (15 time points), and plasma was harvested by centrifugation at $2000 \times \boldsymbol{g}$ at $4{ }^{\circ} \mathrm{C}$ for $10 \mathrm{~min} .{ }^{125} \mathrm{I}$-HSA or ${ }^{125} \mathrm{I}-\mathrm{PEG}-\mathrm{HSA}$ associated radioactivity in the plasma samples was determined after precipitation with $10 \%$ trichloroacetic acid $(\mathrm{TCA})(\mathrm{v} / \mathrm{v}){ }^{16)}$ The radioactivity of the TCA precipitates was counted on the Packard Cobra II Auto-Gamma Counter. Pharmacokinetic parameters were evaluated using practical pharmacokinetic program version 97 (supplied by Chinese Pharmacological Society).

The tissue distribution study of ${ }^{125} \mathrm{I}-\mathrm{HSA}$ and ${ }^{125} \mathrm{I}$-PEG-HSA was also performed in mice by injection into the tail vein. The animals were sacrificed at $1,4,12$, and $24 \mathrm{~h}$ after injection of the radiolabeled proteins and the following tissues were removed: heart, liver, spleen, lung, kidney, muscle and fat. All tissues were then rinsed with saline, weighed and counted for the radioactivity with gamma counter. The contents of ${ }^{125}$ I-labeled proteins in various rat tissues and organs were expressed as the percent of the injected dose per gram of tissue $(\% \mathrm{ID} / \mathrm{g})$, and compared with that in plasma. For plasma, the volume (in milliliters) was used instead of the weight (\%ID/ $\mathrm{mL}) .{ }^{17)}$

Pulmonary Microvascular Permeability of Native and PEGylated HSA LPS-induced capillary leak was used to compare the pulmonary microvascular permeability properties of HSA and PEG-HSA. HSA and PEGylated HSA were labeled with fluorescein isothiocyanate (FITC) as previously described. ${ }^{18)}$ Mice were randomly allocated to three experimental groups: control group (saline+FITC-HSA), HSA treated modeling group (LPS+FITC-HSA), and PEG-HSA treated modeling group (LPS+FITC-PEG-HSA). After Kunming mice were chloral hydrate-anesthetized, $10 \mu \mathrm{g}$ LPS in $50 \mu \mathrm{L}$ saline was administrated intranasally (i.n.) to induce acute lung injury. Control mice were given $50 \mu \mathrm{L}$ saline without LPS as previously described. ${ }^{19)}$ FITC labeled protein $(0.42 \mathrm{mg} / \mathrm{kg})$ were administrated intravenously (i.v.) via a tail vein $20 \mathrm{~min}$ after the injection of LPS or saline. At $4 \mathrm{~h}$ post LPS administration, mice were anesthetized, and the thorax was opened, left atrium incised, and the lung was perfused in situ with isotonic saline via the pulmonary artery. Then the flushed pulmonary tissue were removed, weighed, homogenized and centrifuged, the optical density of the supernatant was determined spectrophotometrically at $490 \mathrm{~nm}$. FITC concentration was calculated against a standard curve, the infiltration of FITC labeled protein in pulmonary tissue per gram was then calculated and expressed as $\mathrm{mmol}$ of protein/g of tissue. For the fluorescence studies, lung sections were examined with inverted fluorescence microscope using $40 \times$ objectives.

Pharmacodynamics of Native and PEGylated HSA in Lipopolysaccharide-Induced Sepsis Model with Capillary Leakage Rats were anaesthetized with an initial intraperitoneal injection of sodium pentobarbital $(60 \mathrm{mg} / \mathrm{kg})$ and maintained by repeated intraperitonea injections of pentobarbital $(20 \mathrm{mg} / \mathrm{kg})$. The body temperature was kept at $37-39^{\circ} \mathrm{C}$ by using a circulating water mattress and warmed solutions. The right carotid artery and left femoral vein were surgically exposed. For lipopolysaccharide (LPS), fluid administration and blood sample collection a venous catheter was inserted into the femoral vein. A tracheotomy was performed by inserting a trachea cannula, an arterial catheter was inserted into the right carotid artery and connected to the pressure transducer. The blood pressure was continuously monitored via BL-420F biological functional experimental system.

Eight animals each were randomized to fluid therapy with PEG-HSA, HSA and saline. LPS-induced sepsis with capillary leakage was used to evaluate the effects of different fluid on maintenance of plasma volume. A $0.1 \mathrm{~mL}$ blood sample was collected from femoral vein for baseline haematocrit (Hct). Mean arterial pressure (MAP) was continuously measured. LPS (from Escherichia coli, serotype O55:B5, Sigma) was given intravenously at $20 \mathrm{mg} / \mathrm{kg}$ of body weigh. ${ }^{20)}$ At $2 \mathrm{~h}$ following LPS injection, PEG-HSA and HSA in saline were administered at $60 \mathrm{mg} / \mathrm{mL}$ (protein concentration) for a dosage of $0.3 \mathrm{~g} / \mathrm{kg}$. MAP monitoring was conducted from prior to LPS injection until $2 \mathrm{~h}$ after PEG-HSA or HSA treatment. Hct measurement was performed at $2 \mathrm{~h}$ post LPS injection and $2 \mathrm{~h}$ post fluid (PEG-HSA, HSA and saline) administration, respectively.

Statistical Analysis All data are expressed as mean \pm S.D., except where otherwise noted. Statistic analysis was performed using $3 P 97$ (Chinese Academy of Sciences Mathematics Research Institute).

\section{RESULTS}

PEGylation of HSA with PEG $_{20 \mathrm{kDa}}$-Mal The activated 


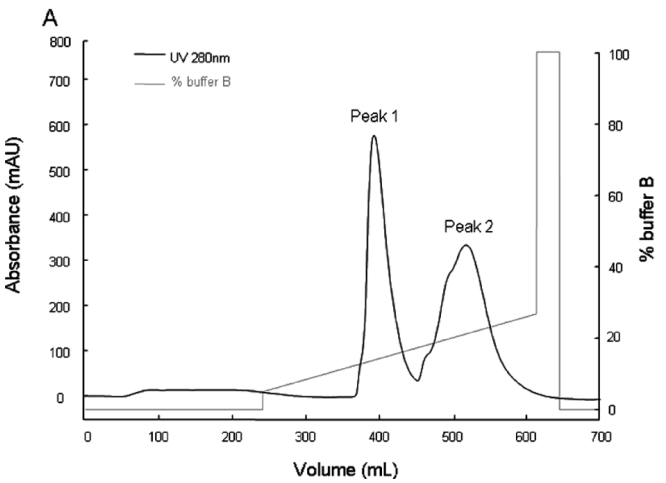

B

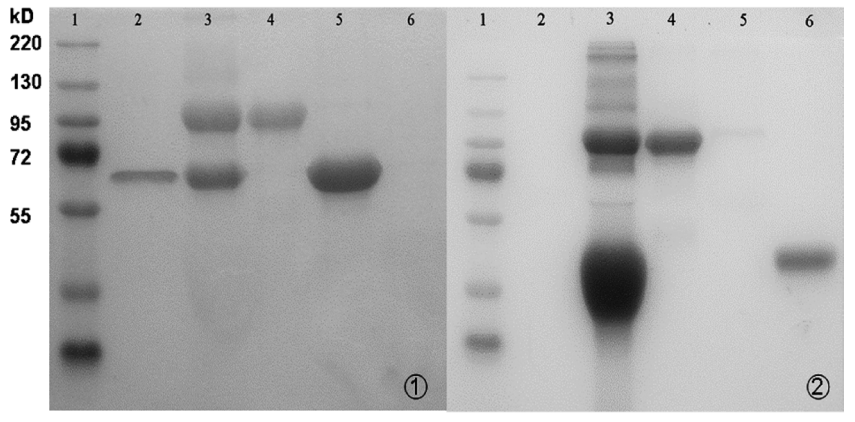

Fig. 1. Purification of the PEGylated HSA

Panel A shows the DEAE Sepharose FF column chromatography of the HSA PEGylation reaction mixture. Peak 1, Cys34 mono-mPEG-HSA conjugate; Peak 2, unreacted HSA. Panel B shows SDS-PAGE of fractions from various column peaks. (1) is gel stained with Coomassie brilliant blue; (2) is gel stained with iodine. lane 1, markers; lane 2, native HSA; lane 3, PEGylation reaction mixture; lane 4, peak 1 eluted from DEAE column, which is monoPEGylated HSA; lane 5, peak 2, which is unmodified HSA; lane 6, $\mathrm{PEG}_{20 \mathrm{kDa}}-\mathrm{Mal}$.

$\mathrm{PEG}_{20 \mathrm{kDa}}$ was used to modify HSA at different conditions as shown in the section of Materials and Methods. Reaction mixtures under different condition were examined by SDS-PAGE. The result showed that compared with the lane of native HSA $(66.5 \mathrm{kDa})$ and the protein molecular weight markers, a new protein band with the apparent molecular weight of about $90 \mathrm{kDa}$ was observed in each reaction mixture lane and few di-PEGylated or tri-PEGylated proteins were detected (Fig. 1B lane 3). This result indicated that PEGylation of HSA molecule was achieved by alkylation of cysteine (sulfhydryl group) with $\mathrm{PEG}_{20 \mathrm{kDa}}$-Mal reagent and only one PEG chain was attached onto each HSA molecule, which means that HSA was monoPEGylated.

The ratios of the optical densities of the PEG-HSA bands and the HSA bands were chosen as the evaluation parameters to assess the modification rates of the products. Taking account into the reaction yields, homogenicity of the products and the production cost, the optimum conditions are as follows: $10 \mathrm{mg} / \mathrm{mL}$ of native HSA, $50 \mathrm{mmol} / \mathrm{L}$ PBS, $\mathrm{pH} 6.5$, containing $10 \mathrm{mmol} / \mathrm{L}$ EDTA, at a protein/PEG molar ratio of $1: 2$ and incubate for $20 \mathrm{~h}$ at $37^{\circ} \mathrm{C}$. At these optimum conditions, the PEGylated protein appeared as single band of about $90 \mathrm{kDa}$ by $10 \%$ SDS-PAGE and the modification yield was over $50 \%$ estimated by optical density scanning of the electrophoretic bands.

Purification of the PEGylated HSA by DEAE Sepharose FF Column Chromatography PEGylated HSA was purified from non-PEGylated protein and unreacted PEG-Mal by DEAE Sepharose column chromatography (Fig. 1A). Fractionation of the HSA PEGylation reaction mixture by DEAE Sepharose column chromatography yielded two major peaks. SDS-PAGE analysis of the column fractions indicated that the early eluting peak (peak 1) was mono-PEGylated HSA, and the later eluting peak (peak 2) was unmodified HSA (Fig. 1B). The result of gel staining with barium iodide and Coomassie brilliant blue showed that neither impure protein nor free PEG was detected in the lane of purified PEG-HSA (peak 1). The purity of HSA conjugate could reach up to $95 \%$ by optical density scanning.

Determination of PEGylation Site Theoretically, there is only one free cystine residue in each HSA molecule and the activated PEG-Mal could specifically and covalently bind to thiol group. Therefore, the activated PEG should specifically

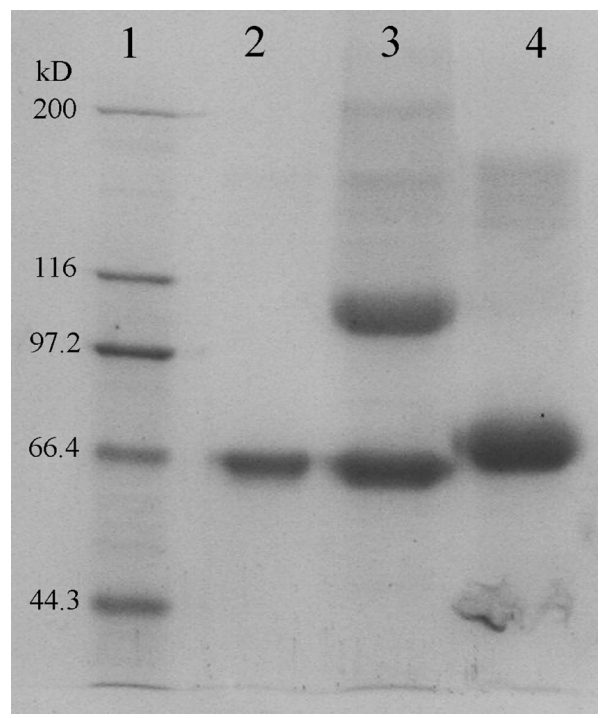

Fig. 2. SDS-PAGE of PEGylation Reaction Mixture and the PEGylation Reaction Mixture Treated with NEM Previously

Lane 1, markers; lane 2, native HSA; lane 3, PEGylation reaction mixture (HSA+PEG-Mal); lane 4, PEGylation reaction mixture treated with NEM previously (HSA+NEM+PEG-Mal). Gels were stained with Coomassie brilliant blue.

attach to Cys34 of the HSA molecule. A two-step method consisting of blockage and PEGylation was applied to determine the modification site. ${ }^{13)}$ SDS-PAGE analysis (Fig. 2) subsequently showed that NEM-treated HSA could no longer be PEGylated by PEG-Mal, thus confirming that PEG-Mal was specifically attached to the unique thiol group of HSA, Cys34.

Secondary Structure Analysis by Far-UV CD Measurement To obtain information on secondary protein structures, CD measurements of HSA and PEG-HSA were performed in the far-UV regions (Fig. 3). Employed the CDNN software, the secondary structures of HSA and PEGHSA were caculated (Table 1). The superimposable spectra indicated that the PEG-HSA structure was almost identical to that of native HSA, which showed that PEGylation didn't induce significant alteration of the secondary protein structure.

Hydrodynamic Radius Determination by Dynamic Light Scattering The distribution of the hydrodynamic radius of HSA and PEG-HSA in saline obtained by DLS is shown in Fig. 4. The calculated $R_{\mathrm{h}}$ of PEG-HSA was $47.25 \mathrm{~nm}$, which 


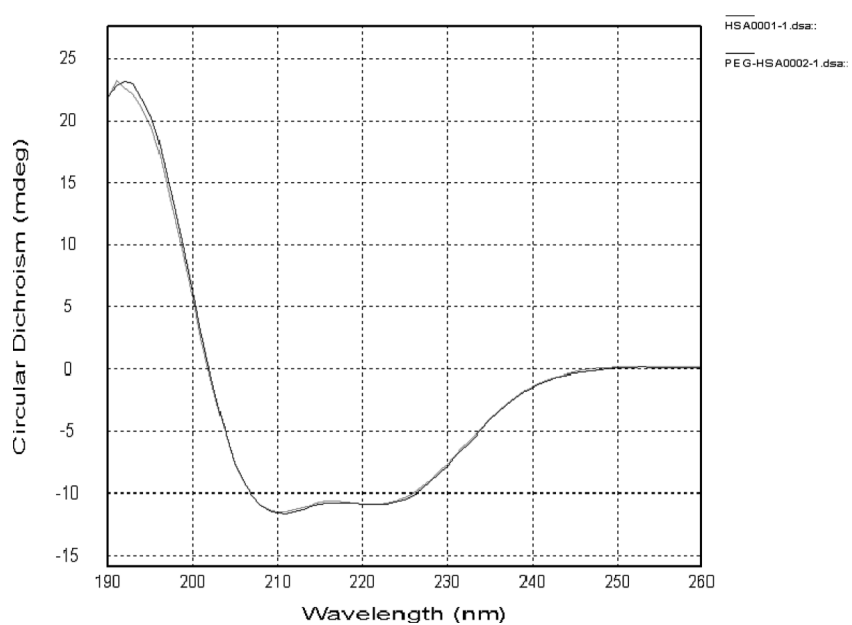

Fig. 3. CD Profile of Native HSA (Dotted Line) and HSA Modified with PEG-Mal (Gray Line)

$\mathrm{CD}$ spectra were recorded at room temperature with a $0.1 \mathrm{~cm}$ light path cuvatte, all samples were solved in $50 \mathrm{mmol} / \mathrm{L}$ phosphate buffer, $\mathrm{pH} 7.4$.

Table 1. Relative Percentage of Conformation of HSA and PEG-HSA on $\mathrm{CD}$

\begin{tabular}{ccccc}
\hline \hline \multirow{2}{*}{ Group } & \multicolumn{4}{c}{ The contents of secondary structures } \\
\cline { 2 - 5 } & $\alpha$-Helix & Parallel & $\beta$-Turn & Random coil \\
\hline HSA & $94.2 \%$ & $0.8 \%$ & $6.5 \%$ & $2.2 \%$ \\
PEG-HSA & $94.6 \%$ & $0.8 \%$ & $6.4 \%$ & $2.1 \%$ \\
\hline
\end{tabular}
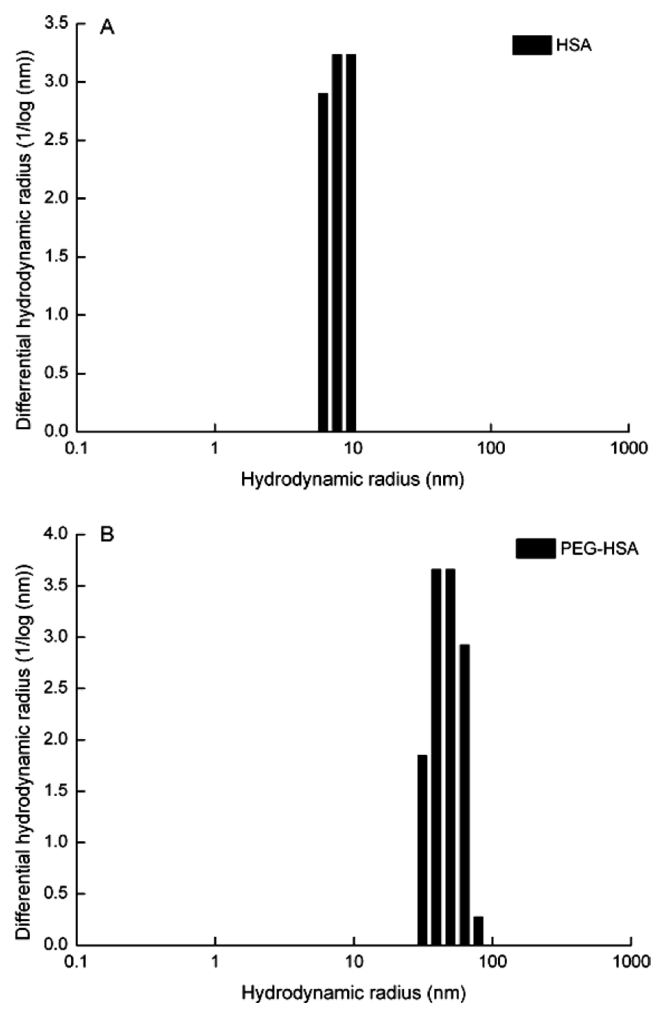

Fig. 4. The Hydrodynamic Radius Distribution of HSA and PEG-HSA with Concentration of $0.01 \mathrm{mg} / \mathrm{mL}$ in Saline at $25^{\circ} \mathrm{C}$

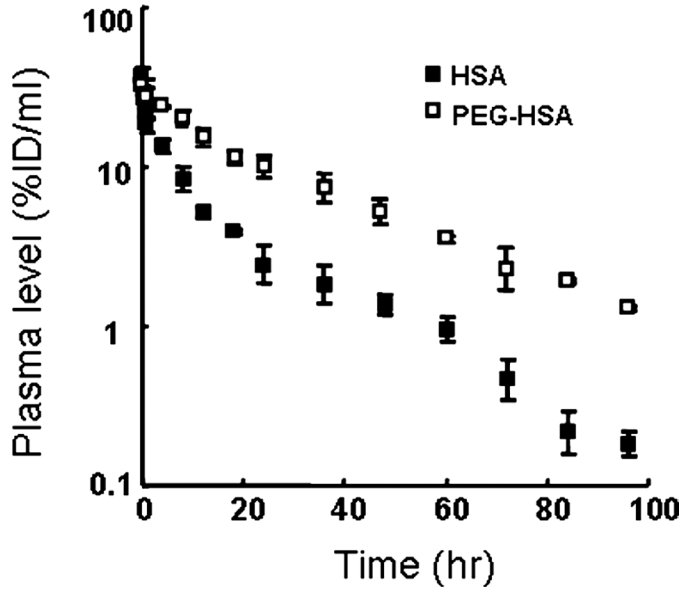

Fig. 5. Plasma Level of Radiolabeled Proteins after a Single Intravenous Administration of ${ }^{125}$ I-HSA (Filled Symbol) or ${ }^{125}$ I-PEG-HSA (Open Symbol) to Normal Mice, Expressed as Percents of Injected Dose per Milliliter Plasma $(\% \mathrm{ID} / \mathrm{mL})$, Each Point Represents the Mean \pm S.D. $(n=3)$, the Injection Dose of HSA and PEG-HSA Were $15.4 \mathrm{MBq} / \mathrm{kg}$ and $5.5 \mathrm{MBq} / \mathrm{kg}$, Respectively

Table 2. Pharmacokinetic Parameters Estimated by Two-Compartmental Model Analysis Following a Single i.v. Bolus Injection of ${ }^{125}$ I-HSA or ${ }^{125}$ I-PEG-HSA to Mice

\begin{tabular}{ccc}
\hline \hline Paramater & HSA & PEG-HSA \\
\hline$A U C_{0-\infty}(\mu \mathrm{g} \cdot \mathrm{h} / \mathrm{mL})$ & $4.81 \pm 0.06$ & $17.05 \pm 0.63^{* *}$ \\
$t_{1 / 2 \alpha}(\mathrm{h})$ & $0.41 \pm 0.05$ & $0.37 \pm 0.08$ \\
$t_{1 / 2 \beta}(\mathrm{h})$ & $9.51 \pm 2.22$ & $21.91 \pm 2.00^{*}$ \\
$C L(\mathrm{~mL} / \mathrm{h})$ & $0.43 \pm 0.03$ & $0.13 \pm 0.003^{* *}$ \\
$M R T(\mathrm{~h})$ & $20.42 \pm 0.96$ & $31.87 \pm 1.55^{*}$ \\
$V d s s(\mathrm{~mL})$ & $5.54 \pm 0.86$ & $4.03 \pm 0.20$ \\
\hline
\end{tabular}

Data are expressed as mean \pm S.D. $(n=3) .{ }^{*} p<0.05, * * p<0.01,{ }^{125}$ I-PEG-HSA $v s$. ${ }^{125}$ I-HSA.

was increased by over 6 times, as compared with that of HSA molecule. These results suggest that the modification of HSA with PEG could significantly increase its hydrodynamic radius. Therefore the larger hydrodynamic radius of PEGHSA may reduce its extravasation and keep it stay longer in vascular.

Pharmacokinetics and Tissue Distribution of Native and PEGylated HSA Figure 5 showed the plasma concentration of ${ }^{125}$ I-HSA and ${ }^{125}$ I-PEG-HSA versus time profiles following a single i.v. bolus injection in mice. The plasma concentration of native HSA and PEG-HSA over time followed exponential decline after i.v. administration. Compared with the native HSA, the elimination of PEG-HSA was slower, which indicated that PEG-HSA possessed longer half-life in the circulation. The pharmacokinetic parameters for the two proteins analyzed by a two-compartment open model were listed in Table 2 . The half-life of the PEG-HSA was significantly prolonged compared with that of the native HSA. In addition, decreased clearance $(C L)$ and increased mean retention time $(M R T)$ were observed as well. These all suggested an improvement in the retention of ${ }^{125}$ I-PEG-HSA in the circulation.

The tissue distribution of ${ }^{125} \mathrm{I}$-HSA or ${ }^{125} \mathrm{I}$-PEG-HSA in mice was shown in Fig. 6. The concentration of PEG-HSA was significantly higher than that of HSA in blood at $1,4,12$ and $24 \mathrm{~h}$, which confirmed the improved retention of PEG- 


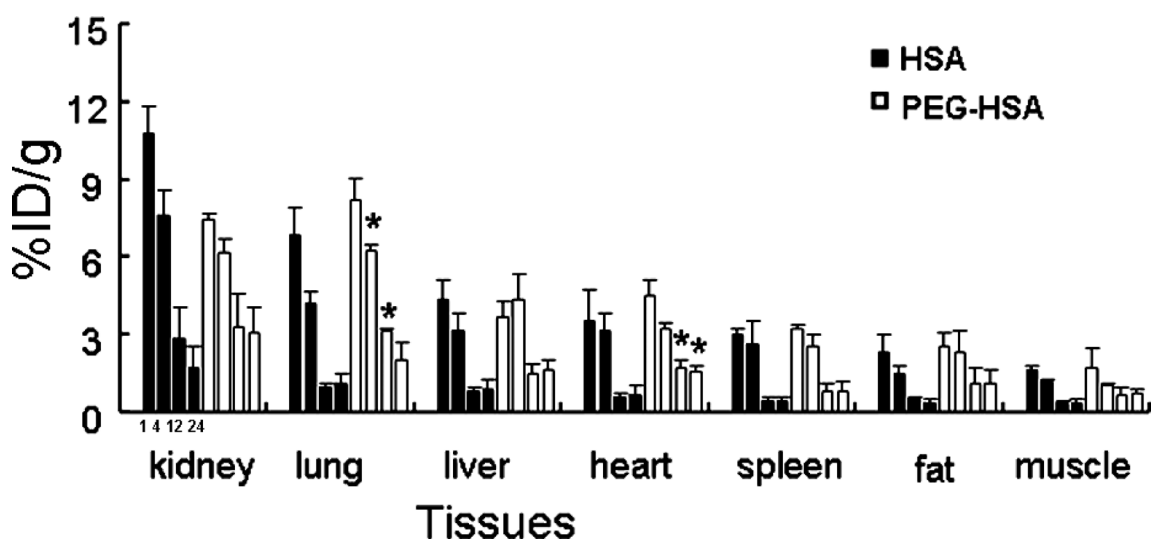

Fig. 6. Tissue Distribution of HSA (Filled Bar) and PEG-HSA (Open Bar) at 1, 4, 12, and 24h Time Points in Normal Mice after a Single Intravenous Administration

Data are expressed as mean \pm S.D. of percents of the injected dose per gram tissue $(\% \mathrm{ID} / \mathrm{g})$ with 3 mice per group, the injection dose of HSA and PEG-HSA were $15.4 \mathrm{MBq} / \mathrm{kg}$ and $5.5 \mathrm{MBq} / \mathrm{kg}$, respectively. ${ }^{*} p<0.05$, PEG-HSA treated group $v_{s}$. HSA treated group in the same tissue at the same time point.
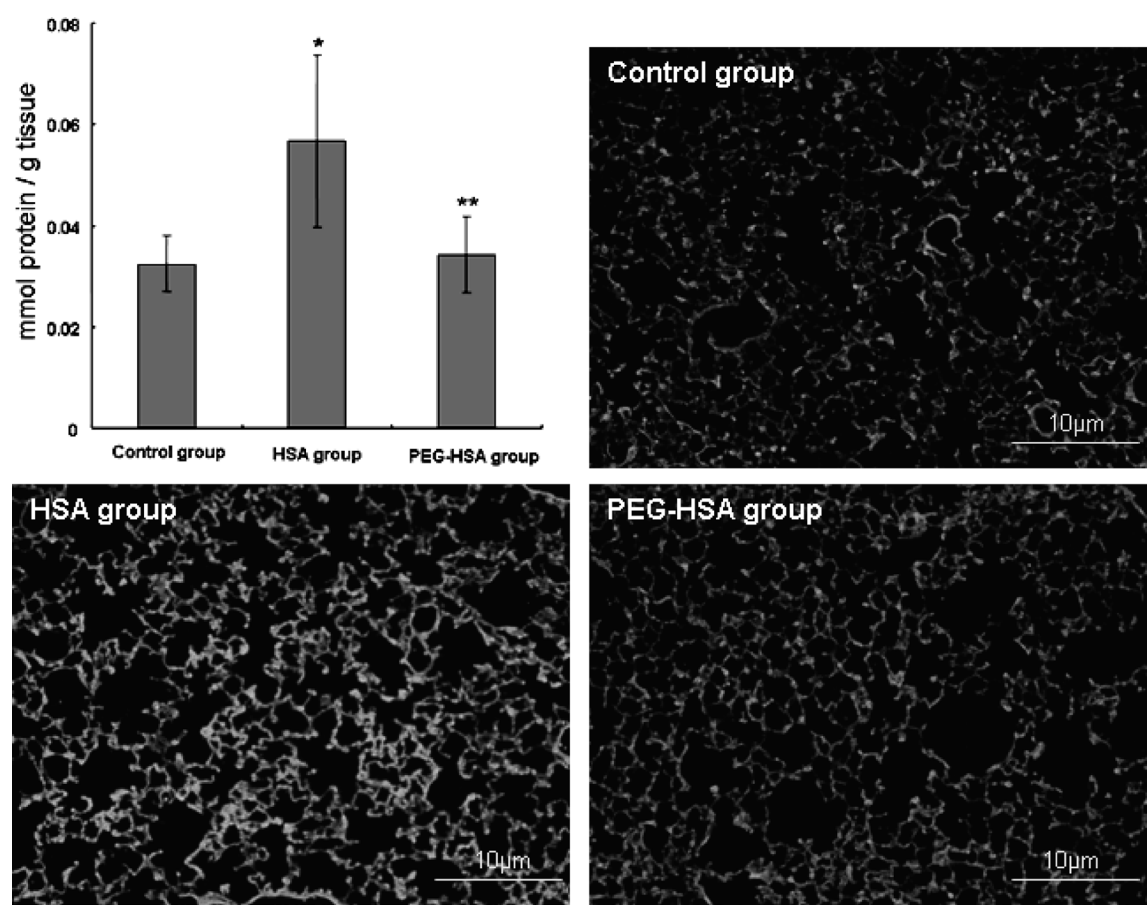

Fig. 7. Protein Concentration of HSA and PEG-HSA in Pulmonary Tissues (Left Upper) and Fluorescence Micrographs of Pulmonary Tissue at $4 \mathrm{~h}$ after LPS Intranasal Administration in Acute Lung Injury Mice

Data are expressed as mean \pm S.D. of mmol/L of protein/g of tissue with 4 mice per group. ${ }^{*} p<0.05$, LPS + FITC-HSA treated group $v s$. saline (control group). ${ }^{* *} p<0.05$, LPS+FITC-PEG-HSA treated group $v$. LPS+FITC-HSA treated group.

HSA in plasma. PEG-HSA exhibited no significant difference in tissue tropism with respect to HSA. Both ${ }^{125} \mathrm{I}-\mathrm{HSA}$ and ${ }^{125}$ I-PEG-HSA underwent a wide distribution in the various tissues and organs throughout the body. Radioactivity was mainly distributed to highly perfused kidneys and lung, and to a lesser extent to the liver and heart, with other organs showing low signals. At 12 and $24 \mathrm{~h}$ postdosing, the radioactivity of PEG-HSA in the various tissues and organs was generally higher than that of HSA, especially in lung and heart.

Pulmonary Microvascular Permeability of Native and PEGylated HSA The vascular permeability of the native and PEGylated HSA was evaluated using LPS-induced acute lung injury as a model system. In this study, the pulmonary capillary leak was confirmed by the increase of HSA escap- ing into the extravascular space in the HSA treated modeling group (Fig. 7). The result showed that the effusion into the lung parenchyma of native HSA and PEGylated HSA was separately $0.057 \pm 0.017$ and $0.034 \pm 0.007 \mathrm{mmol}$ protein per gram tissue. Under fluorescent microscope, a lower vascular permeability of the FITC-PEG-HSA was also observed compared with that of FITC-HSA. These results suggested that the PEGylation of HSA could reduce the extravasation into lung parenchyma.

Pharmacodynamics of Native and PEGylated HSA in Lipopolysaccharide-Induced Sepsis Model with Capillary Leakage Levels of Hct and MAP pre and after treatment are given in Table 3. During sepsis there was a significant decrease in MAP in all groups. The MAP recovery in the PEG- 
Table 3. Mean Arterial Pressure (MAP) and Haematocrit in the Three Groups at Baseline, $2 \mathrm{~h}$ Post LPS Administration and $2 \mathrm{~h}$ Post Different Fluid (Saline, HSA and PEG-HSA) Treatment

\begin{tabular}{|c|c|c|c|}
\hline & Baseline & $2 \mathrm{~h}$ Post LPS infusion & $2 \mathrm{~h}$ Post fluid treatment \\
\hline \multicolumn{4}{|l|}{ MAP (mmHg) } \\
\hline Saline & $144 \pm 4$ & $94 \pm 10 * *$ & $95 \pm 18$ \\
\hline HSA & $135 \pm 8$ & $92 \pm 6^{* *}$ & $98 \pm 15$ \\
\hline PEG-HSA & $143 \pm 13$ & $92 \pm 12 * *$ & $117 \pm 7^{\#, \$}$ \\
\hline \multicolumn{4}{|l|}{ Het $(\%)$} \\
\hline Saline & $47.2 \pm 3.2$ & $51.7 \pm 3.0 *$ & $43.0 \pm 2.4^{\#}$ \\
\hline HSA & $46.9 \pm 2.1$ & $50.7 \pm 2.6^{* *}$ & $41.4 \pm 3.6^{\#}$ \\
\hline PEG-HSA & $46.7 \pm 2.2$ & $49.9 \pm 2.9 * *$ & $38.0 \pm 3.0^{\#, \$}$ \\
\hline
\end{tabular}

Data are expressed as mean \pm S.D. $(n=8) .{ }^{*} p<0.05,{ }^{* *} p<0.01,2 \mathrm{~h}$ post LPS infusion $v s$. baseline. ${ }^{*} p<0.01,2 \mathrm{~h}$ post fluid treatment $v s .2 \mathrm{~h}$ post LPS infusion. ${ }^{\$} p<0.05$, PEG-HSA vs. saline, HSA.

HSA group $2 \mathrm{~h}$ after fluid treatment was better than in saline and HSA groups. MAP recovery in HSA and saline treated rats were similar. Hct after LPS injection was significantly higher than baseline, which indicated a lower plasma volume. At $2 \mathrm{~h}$ following fluid treatment, effects of hemodilution were observed in all rats at different degree. Moreover, Hct was much lower in PEG-HSA group than HSA and saline groups, suggesting a relatively greater increment in intravascular plasma volume. The resuscitation effect of PEG-HSA is better than that of HSA and saline in LPS-induced sepsis with systemic capillary leakage.

\section{DISCUSSION}

HSA is a protein of therapeutic interest in the treatment of hypovolemia caused by traumatic shock, massive hemorrhage, burns, cirrhosis with ascites and hypoalbuminemia, with usual dosage of $10 \mathrm{~g} / \mathrm{dose},{ }^{4)}$ but limited amounts originating from human plasma are available in clinical use. Although gene manipulation techniques were thought to be a potential approach to develop an ideal candidate for HSA, it still faces many obstacles in the large-scale production of pharmaceutical grade rHSA.

As a nonimmunogenic and biocompatible water-soluble polymer, PEG was widely used in protein conjugation and low molecular weight drug conjugation. ${ }^{21)}$ PEG conjugation is regarded as a valuable technique in applied biotechnology that makes protein drugs more water-soluble, non-aggregating, non-immunogenic and more stable to proteolytic digestion. ${ }^{22,23)}$ The most common method for PEGylation attachs PEG to amine groups in proteins, typically at the $\varepsilon$-amino group of surface lysine residues or the $\mathrm{N}$-terminal amino acid residues. ${ }^{24)}$ However, HSA contains 58 lysine residues. Thus, random polymer conjugation yields inhomogeneous derivatives with undefined composition, significant loss in biological activity and unpredictable in vivo behavior. ${ }^{25)}$ In order to overcome these limitations, site-specific PEGylation which generates well-defined, specifically labeled proteins with minimal effects on receptor binding or substrate recognition have been developed. ${ }^{26)}$ Amino acids seldom present in the protein structure are natural sites for selective PEGylation. HSA molecule contains only one free thiol at Cys $34,{ }^{2)}$ which offer a target for site-specific attachment with thiol reactive maleimide. In our current study, we modified HSA in a site-specific manner by covalently conjugation of commercial $\mathrm{PEG}_{20 \mathrm{kDa}}-\mathrm{Mal}$ to Cys34 of the HSA molecule. The structurally uniform PEG-HSA was easy to obtain and the preparation method was reproducible. PEG-Mal with a large molecular weight of $20 \mathrm{kDa}$ was selected in order to endow the protein with marked masking effect and a larger hydrodynamic radius. In our study, the hydrodynamic radius of PEG-HSA was increased by over 6 times. The state of Cys34 is a possible site of dimerization, thereby PEGylation at Cys34 could also be helpful to prevent the heterogeneity in albumin. Crystallographic studies of albumin show that Cys34 is buried in a shallow crevice, $9.5 \AA$ deep. ${ }^{27)}$ This may explain why the modification yield at Cys34 was only about $50 \%$. The attachment of PEG was proved to be selective to the unique thiol group of HSA by a two-step method in this study. SDS-PAGE was used to examine the distribution and the molecular weight of PEGylation product. We observed that the PEGylated HSA migrated at a larger apparent molecular weight than the sum of the molecular weights of both HSA and PEG. This decreased electrophoretic mobility was caused by the protein PEGylation, which had been previously observed by Kurfürst. ${ }^{12)}$

It is well known that increased microvascular permeability is an important pathophysiological alteration in diseases such as burn injury, sepsis and trauma and so on. For example, patients suffering from general inflammation, e.g. after trauma and surgery, often develop increased microvascular permeability. This may result in transcapillary leakage and hypovolemia. ${ }^{28)}$ Under this condition, correction of low plasma volume therefore may be essential to maintain adequate organ perfusion and oxygen delivery. ${ }^{29)}$ For decades there has been debates regarding which kind of plasma expander should be infused. Crystalloids, due to small molecular weight, could pass freely across the capillary membrane, so their plasma expanding effect is transit. Colloid solution such as albumin with larger molecular weight could effuse at a more steady speed, hence the retention in vascular of colloid solution is increased and the normovolemia could be maintained for longer. However, according to the modern two-pore theory of transvascular exchange, ${ }^{30)}$ transcapillary leakage of macromolecules occurs through the large pores of the capillary membrane. HSA infused would continuously permeate into organs and cleared from the circulation. According to the previous research, ${ }^{31)}$ albumin diffused through pores with an effective equivalent pore radius of $145 \AA$. So the extravasation of proteins from the circulation is primarily determined by molecular dimension. Researchers conjectured that increasing the molecular size of albumin by fusion of two HSA molecules to stop leakage to extravascular spaces may be a potential solu- 
tion. The result showed that the biological half-life the rHSA dimer was approximately 1.5 times greater and the vascular permeability of the rHSA dimer was less than that of the native HSA in paw edema mouse models. ${ }^{8}$ In contrast to their observations, a previous study showed that a covalently linked recombinant albumin dimer was more rapidly cleared in vivo than wild-type albumin, ${ }^{32)}$ they contributed this to the role for the reticuloendothelial system in the differential clearance of the larger protein. The present study evaluated the pharmacokinetics of PEG-HSA in a mouse system, the results showed that plasma half-life of modified HSA was $21.91 \pm 2.00 \mathrm{~h}, 2.3$ times greater than the native protein. It has been reported that the mechanism of HSA breakdown involved uptake into endocytotic vesicles, which could fuse with lysosomes in endothelial cells. This process involves binding to the scavenger receptors (gp18 and gp30) on the endothelial surface membrane. ${ }^{33)}$ Hence, it is likely that chemical modification reduces degradation, the masking effect of PEG attached to the surface of HSA molecule could prevent the recognization of the scavenger receptors, reticuloendothelial system and proteolytic enzymes. Tissue distribution study showed that in the kidney, the concentration of HSA is relatively higher than that of PEG-HSA, especially at 1 and $4 \mathrm{~h}$ time points after intravenous administration. This consequence may be caused by the relatively small hydrodynamic radius of HSA and the unique physiological structure of kidney. Compared with capillaries in other tissues, the blood pressure of glomerular capillaries is higher and the total surface area is larger, as blood flows through renal glomerulus, HSA with relatively smaller molecular weight tends to pass through the fenestrae and distribute into kidney more easily than PEG-HSA.

Under normal physiological conditions, HSA molecule distributes between the intravascular and extravascular compartments. Each day, $120-145 \mathrm{~g}$ of HSA is lost into the extravascular space and most of this is recovered back into the circulation by lymphatic drainage. ${ }^{6}$ Critical illness, such as sepsis, shock and major injury, breaks the distribution balance of HSA between the two compartments. The altered distribution is related to an increase in capillary leakage, the normal transcapilliary rate for albumin increases by up to 3 times in patients with septic shock and by 1 time after cardiac surgery. ${ }^{34)}$ It involves the dysfunction of the endothelial barrier, resulting in capillary leakage and loss of protein and large volumes of fluid. ${ }^{6)}$ In this situation, using exogenous albumin to increase the plasma albumin concentration seems beneficial, but it is argued that the infused albumin was inefficient in reducing liquid shift and may contribute to delayed pulmonary oedema. ${ }^{35)}$ In this study, we expect that increasing the molecular size of albumin by PEG modification could reduce the flow of albumin across capillary membranes under the conditions with increased capillary leakage.

LPS-induced acute lung injury was used to compare the pulmonary microvascular permeability properties of HSA and PEG-HSA. In this model, inflammatory cascading reactions induce increased microvascular permeability and capillary leakage, especially in pulmonary tissue. ${ }^{36)}$ The lung microvascular permeability was reported to increase significantly at $4 \mathrm{~h}$ after LPS injection. ${ }^{18)}$ We observed a significant lower vascular permeability of the FITC-PEG-HSA at $4 \mathrm{~h}$ after injection, which indicated that increasing the molecular size of albumin by PEG modification could reduce transcapillary loss of albumin molecules. Lipopolysaccharide administration has often been used for the experimental induction of septic shock in laboratory animals. ${ }^{20}$ Increased vascular permeability is pathogenic for capillary leak syndrome (CLS). ${ }^{37)}$ In our study haematocrit increased in all groups after LPS infusion, this is a characteristic of capillary leak. After LPS-induced septic shock, better recovery in blood pressure and haemodilution was observed in rats treated with PEG-HSA. Furthermore, the superior resuscitation effect of PEG-HSA was still distinct $2 \mathrm{~h}$ after fluid infusion, which indicated longer intravascular retention in rats. Based on the hydrodynamic radius determination and pulmonary microvascular permeability measurement, it can be inferred that increasing the molecular weight of HSA by PEGylation may contribute to the improvement of the intravascular retention, prevent intravascular fluid loss and the interstitial liquid accumulation.

In summary, this study provides evidences that it is possible, through a simple procedure involving thiol-specific PEG-Mal conjugation, to improve the half-life in the circulation and reduce the vascular permeability of HSA infused, and consequently promote the intravascular retention and decrease the administration frequency. The PEGylated HSA is a superior candidate in treating capillary permeability increase related illness because of its longer biological half-life and good intravascular retention. The our future work would focus on the optimization of the modification strategy including the molecule weights, shapes and the active groups of different PEG agents and different modification sites. In addition, tolerability and the safety in vivo of different modified products will also be our main task.

Acknowledgement This study was funded by a Grant from Department of Science and Technology of Shandong Province (No. 2010GSF10215).

\section{REFERENCES}

1) Minghetti PP, Ruffner DE, Kuang WJ, Dennison OE, Hawkins JW, Beattie WG, Dugaiczyk A. Molecular structure of the human albumin gene is revealed by nucleotide sequence within q11-22 of chromosome 4. J. Biol. Chem., 261, 6747-6757 (1986).

2) Christodoulou J, Sadler PJ, Tucker A. A new structural transition of serum albumin dependent on the state of Cys34. Detection by 1H-NMR spectroscopy. Eur. J. Biochem., 225, 363-368 (1994).

3) Doweiko JP, Nompleggi DJ. Role of albumin in human physiology and pathophysiology. JPEN J. Parenter. Enteral Nutr., 15, 207-211 (1991).

4) Kobayashi K. Summary of recombinant human serum albumin development. Biologicals, 34, 55-59 (2006).

5) Christ F, Gamble J, Gartside IB, Kox WJ. Increased microvascular water permeability in patients with septic shock, assessed with venous congestion plethysmography (VCP). Intensive Care Med., 24, $18-27$ (1998).

6) Nicholson JP, Wolmarans MR, Park GR. The role of albumin in critical illness. Br. J. Anaesth., 85, 599-610 (2000).

7) Marx G. Fluid therapy in sepsis with capillary leakage. Eur. J. Anaesthesiol., 20, 429-442 (2003).

8) Matsushita S, Chuang VT, Kanazawa M, Tanase S, Kawai K, Maruyama T, Suenaga A, Otagiri M. Recombinant human serum albumin dimer has high blood circulation activity and low vascular permeability in comparison with native human serum albumin. Pharm. Res., 23, 882-891 (2006).

9) Bonora GM, Ivanova E, Zarytova V, Burcovich B, Veronese FM. 
Synthesis and characterization of high-molecular mass polyethylene glycol-conjugated oligonucleotides. Bioconjug. Chem., 8, 793-797 (1997).

10) Inada $Y$, Furukawa M, Sasaki H, Kodera $Y$, Hiroto M, Nishimura $\mathrm{H}$, Matsushima A. Biomedical and biotechnological applications of PEG- and PM-modified proteins. Trends Biotechnol., 13, 86-91 (1995).

11) Li JW, Xiao NG, Yu RY, Yuan MX, Chen LR, Chen YH, Chen LT. Experimental Principles and Methods of Biochemistry. Peking University Publishers, Beijing (2000).

12) Kurfürst MM. Detection and molecular weight determination of polyethylene glycol-modified hirudin by staining after sodium dodecyl sulfate-polyacrylamide gel electrophoresis. Anal. Biochem., 200, 244-248 (1992).

13) Cantin AM, Woods DE, Cloutier D, Dufour EK, Leduc R. Polyethylene glycol conjugation at Cys232 prolongs the half-life of alphal proteinase inhibitor. Am. J. Respir. Cell Mol. Biol., 27, $659-665$ (2002).

14) Kathmann EEL, Davis DAD, McCormick CL. Water-Soluble Polymers. 60. Synthesis and solution behavior of terpolymers of acrylic acid, acrylamide, and the zwitterionic monomer 3-[(2-acrylamido-2-methylpropyl)dimethylammonio]-1-propanesulfonate. Macromolecules, 27, 3156-3161 (1994).

15) Fraker PJ, Speck JC Jr. Protein and cell membrane iodinations with a sparingly soluble chloroamide, 1,3,4,6-tetrachloro-3a,6a-diphrenylglycoluril. Biochem. Biophys. Res. Commun., 80, 849-857 (1978).

16) Cho HM, Rosenblatt JD, Kang YS, Iruela-Arispe ML, Morrison SL, Penichet ML, Kwon YG, Kim TW, Webster KA, Nechustan H, Shin SU. Enhanced inhibition of murine tumor and human breast tumor xenografts using targeted delivery of an antibody-endostatin fusion protein. Mol. Cancer Ther., 4, 956-967 (2005).

17) Liu J, Zhao T, Tan H, Cheng Y, Cao J, Wang F. Pharmacokinetic analysis of in vivo disposition of heparin-superoxide dismutase. Biomed. Pharmacother., 64, 686-691 (2010).

18) Monsigny M, Roche AC, Midoux P. Uptake of neoglycoproteins via membrane lectin(s) of L1210 cells evidenced by quantitative flow cytofluorometry and drug targeting. Biol. Cell, 51, 187-196 (1984).

19) Szarka RJ, Wang N, Gordon L, Nation PN, Smith RH. A murine model of pulmonary damage induced by lipopolysaccharide via intranasal instillation. J. Immunol. Methods, 202, 49-57 (1997).

20) Ando H, Takamura T, Ota T, Nagai Y, Kobayashi K. Cerivastatin improves survival of mice with lipopolysaccharide-induced sepsis. J. Pharmacol. Exp. Ther., 294, 1043 -1046 (2000).

21) Liu XM, Quan LD, Tian J, Laquer FC, Ciborowski P, Wang D. Syntheses of click PEG-dexamethasone conjugates for the treatment of rheumatoid arthritis. Biomacromolecules, 11, 2621-2628 (2010).

22) Veronese FM, Pasut G. PEGylation, successful approach to drug delivery. Drug Discov. Today, 10, 1451-1458 (2005).

23) Eto $Y$, Yoshioka $Y$, Ishida $T$, Yao $X$, Morishige $T$, Narimatsu S, Mizuguchi H, Mukai Y, Okada N, Kiwada H, Nakagawa S.
Optimized PEGylated adenovirus vector reduces the anti-vector humoral immune response against adenovirus and induces a therapeutic effect against metastatic lung cancer. Biol. Pharm. Bull., 33, $1540-1544$ (2010).

24) Kato K, Tamaki N, Saito $Y$, Fujimoto $T$, Sato A. Amino group PEGylation of bovine lactoferrin by linear polyethylene glycol- $p$ nitrophenyl active esters. Biol. Pharm. Bull., 33, 1253-1255 (2010).

25) Salmaso S, Semenzato A, Bersani S, Mastrotto F, Scomparin A, Caliceti P. Site-selective protein glycation and PEGylation. Eur. Polym. J., 44, 1378-1389 (2008).

26) Basu A, Yang K, Wang M, Liu S, Chintala R, Palm T, Zhao H, Peng P, Wu D, Zhang Z, Hua J, Hsieh MC, Zhou J, Petti G, Li X, Janjua A, Mendez M, Liu J, Longley C, Zhang Z, Mehlig M, Borowski V, Viswanathan M, Filpula D. Structure-function engineering of interferon-beta-1b for improving stability, solubility, potency, immunogenicity, and pharmacokinetic properties by siteselective mono-PEGylation. Bioconjug. Chem., 17, 618-630 (2006)

27) Stewart AJ, Blindauer CA, Berezenko S, Sleep D, Tooth D, Sadler PJ. Role of Tyr84 in controlling the reactivity of Cys34 of human albumin. FEBS J., 272, 353-362 (2005).

28) Dubniks M, Persson J, Grände PO. Effect of blood pressure on plasma volume loss in the rat under increased permeability. Intensive Care Med., 33, 2192-2198 (2007).

29) Haupt MT, Gilbert EM, Carlson RW. Fluid loading increases oxygen consumption in septic patients with lactic acidosis. Am. Rev. Respir. Dis., 131, 912-916 (1985).

30) Rippe B, Haraldsson B. Transport of macromolecules across microvascular walls: the two-pore theory. Physiol. Rev., 74, 163-219 (1994).

31) Sejrsen P, Paaske WP, Henriksen O. Capillary permeability of 131I-albumin in skeletal muscle. Microvasc. Res., 29, 265-281 (1985).

32) McCurdy TR, Gataiance S, Eltringham-Smith LJ, Sheffield WP. A covalently linked recombinant albumin dimer is more rapidly cleared in vivo than are wild-type and mutant C34A albumin. J. Lab. Clin. Med., 143, 115-124 (2004).

33) Schnitzer JE, Bravo J. High affinity binding, endocytosis, and degradation of conformationally modified albumins. Potential role of gp30 and gp18 as novel scavenger receptors. J. Biol. Chem., 268, $7562-7570$ (1993).

34) Fleck A, Raines G, Hawker F, Trotter J, Wallace PI, Ledingham IM, Calman KC. Increased vascular permeability: a major cause of hypoalbuminaemia in disease and injury. Lancet, 1, 781-784 (1985).

35) Manelli JC. Is albumin administration useful in critical care for burnt patients? Ann. Fr. Anesth. Reanim., 15, 507-513 (1996).

36) Groeneveld AB, Bronsveld W, Thijs LG. Hemodynamic determinants of mortality in human septic shock. Surgery, 99, 140-153 (1986).

37) Traber DL. Microvascular leakage in sepsis. Crit. Care Med., 28, $882-883(2000)$ 